\title{
Strategies to change body composition in older adults: do type of exercise and dose distribution matter?
}

\author{
Cristina BLASCO-LAFARGA 1,2, Pablo MONTEAGUDO 1, 3 *, \\ Ainoa ROLDÁN ${ }^{1,2}$, Ana CORDELLAT ${ }^{1,2}$, Caterina PESCE ${ }^{4}$
}

1Sport Performance and Physical Fitness Research Group (UIRFIDE), University of Valencia, Valencia, Spain; 2 Physical Education and Sports Department, University of Valencia, Valencia, Spain; ${ }^{3}$ Department of Education and Specific Didactics, Jaume I University, Castellon, Spain; ${ }^{4}$ Department of Movement, Human and Health Sciences, Foro Italico University, Rome, Italy.

*Corresponding author: Pablo Monteagudo, Sport Performance and Physical Fitness Research Group (UIRFIDE), University of Valencia, C/ Gascó Oliag 3, 46010 Valencia, Spain. E-mail: pablo.monteagudo@uv.es

\section{A B S T R A C T}

BACKGROUND: This study aims to analyze changes in sedentary elderlies' body composition, comparing the impact of two dose distributions: continuous (60 min/session) $v s$. accumulated ( $30 \mathrm{~min} / \mathrm{session}$ in the morning and $30 \mathrm{~min} / \mathrm{session}$ in the afternoon), on two types of physical exercise programs (multicomponent-training [MCT] vs. walking interval-training [WIT]).

METHODS: Forty-six sedentary overweight elderly ( $71.3 \pm 4.3$ years) were evaluated by bioimpedance with a pre-post $2 \times 2$ factorial design. Participants were distributed in four homogeneous groups, considering gait and health parameters.

RESULTS: The overall sample showed significant improvements in fat mass ( $\%$ and $\mathrm{kg})$ and fat-free mass $(\mathrm{kg})$ following 15 weeks of training. There was a main effect of "type of exercise" and a moderating effect of "dose distribution" reflected in the interaction of both factors. Bonferroni analyses of the type*dose interaction revealed significant improvements in all groups, except for MCT-accumulated, on both body composition compartments.

CONCLUSIONS: Starting exercise training, properly periodized and supervised by physical-education trainers, improves body composition of sedentary elderlies regardless of the type of exercise. Accumulated strategies were beneficial only when applied to WIT. Although MTC training improves body composition with only 2 days/week, WIT-accumulated could be a key element in the design of weight control policies looking for breaking sedentary behaviors.

(Cite this article as: Blasco-Lafarga C, Monteagudo P, Roldán A, Cordellat A, Pesce C. Strategies to change body composition in older adults: do type of exercise and dose distribution matter? J Sports Med Phys Fitness 2020;60:000-000. DOI: 10.23736/S0022-4707.20.10321-9)

KEY WORDS: Aging; Sedentary behavior; Exercise; Preventive health services; Aged.

$\mathrm{W}$ ithin the last years, there is an extensive debate about the best approaches to comprehensive tackling the deleterious effects of aging. Body composition emerges as a central issue. Age-related changes such as the decrease in muscle mass, the increase in body fat ${ }^{1}$ and the loss of bone mass ${ }^{2}$ have important systemic implications and are significant contributors to functional limitation in the old age. ${ }^{3,4}$ Physical activity (PA) and physical exercise (PE) are also key factors (where PE it exclusively refer to structured PA purposely design to fitness training). Despite the overwhelming evidence supporting the positive role of
$\mathrm{PE} / \mathrm{PA}$ in managing many of the bad conditions associated with age (including body composition ${ }^{5,6}$ ), getting elderly people physically active is still a challenge. ${ }^{7}$ In this scenery, public health promoters need to solve some scientific inconsistencies, for example, with regard to the possible benefits from segmenting exercise doses (continuous $v s$. accumulative exercise training) $; 8$ or with regard to the influence of the type of exercise on sedentary elderlies' body composition. ${ }^{9}$

In order to alter body composition, elders are required to sustain exercise up to a certain demand, following the 
prescription criteria of frequency, intensity and duration by healthcare institutions. ${ }^{10}$ For example, 30 -min daily of moderate-to-vigorous $\mathrm{PA}$ is associated with a reduced risk of severe sarcopenia and sarcopenic obesity. ${ }^{11}$ But there is the alternative of performing bouts of $>10$ min of moderate-to-vigorous PA to accumulate exercise throughout the day and counteract sedentary behaviour, ${ }^{12}$ with similar results to continuous exercise. ${ }^{8}$ Both, PE and accumulated bouts of PA in sedentary breaks ( $<10 \mathrm{~min}$ ) have shown to attenuate glucose and insulin response, and post prandial lipids, in overweight and obese adults, thus contributing to reduce the cardiometabolic diseases and premature mortality associated to sedentary behaviors. ${ }^{13}$ Moreover, some studies stand out that more breaks within sedentary time are associated with benefits in metabolic risk, particularly in adiposity measures. ${ }^{14}$ These findings suggest that the manner in which sedentary time is accumulated is more important than the total amount of sedentary time.

Since sedentary behaviors increase throughout the course of the day and peak in the evening, PA interventions may need to modify the above cited sedentary pattern, aiming to maintain PA in the morning and reduce the sedentary behavior in the afternoon. ${ }^{15}$ Accumulative exercise (e.g. distributing exercise training in the morning and in the afternoon) could be a good strategy to counteract and reduce this sedentary time, especially in the less active time-slots. Notwithstanding, little is known about whether accumulative proposals convey some advantage compared to similar doses of continuous exercise in sedentary elderlies' body composition. And it remains also unknown whether accumulated training enhances (or not), the benefits from some of their most effective exercise programs (i.e. the widespread cardiovascular walking programs [WP] and the multicomponent training [MCT] programs).

Concerning the type of exercise, walking is the type of activity most often chosen by seniors, 16 in addition to being economical and providing opportunities for company and social support. ${ }^{17}$ WP are effective to increase daily PA $^{18}$ and health-related quality of life, ${ }^{17}$ to reduce risk factors for cardiovascular diseases, 19,20 and to prevent cognitive decline in healthy older adults. ${ }^{21}$ Even so, it remains unclear its impact in sedentary older adults or with mobility limitations, specifically with regard to body composition. In fact, only three studies in the meta-analysis by Murtagh et al. ${ }^{20}$ include elderly close or over the age of 70 .

Similarly, MCT emerges as a powerful alternative to those traditional walking or resistance training programs, particularly due to its high impact on functional and neuro-psychological outcomes in older adults. ${ }^{9}, 22$ MCT com- bines aerobic and strength, with balance and sometimes cognitive tasks, to reap multiple benefits along with a multisystemic physical activation. ${ }^{9}, 23$ Similar to WP, we need to know more about the impact of MCT on body composition, and there is little knowledge with regard of the consequences of accumulated strategies under this neuromuscular approach.

Summarizing, despite intrinsic differences in their frequency, volume and intensity prescription, both types of exercise (i.e., MCT and WP) improve the elderly's functionality, ${ }^{22}$ with some inconsistencies with regard to body composition. In addition, there is scarcity of studies focusing on different dose strategies in training (i.e., continuous $v s$. accumulated) in this population. Therefore, the present study aims to analyze changes in body composition in a group of sedentary elderly, comparing the impact of two different dose distributions: continuous (CON: 60 $\mathrm{min} / \mathrm{session}$ ) vs. accumulated (ACC: $30 \mathrm{~min} / \mathrm{session}$ in the morning and $30 \mathrm{~min} / \mathrm{session}$ in the afternoon), on the efficacy of two different PE training programs: a walking interval-training program (WIT) and a MCT program called EFAM-UVO 24 Whether one dose distribution, type of exercise, or a specific combination of both, is the best strategy to improve the body composition, is an important health issue in this population.

\section{Materials and methods}

\section{Experimental procedure and participants}

This quasi-experimental and longitudinal study was carried out with a $2 \times 2$ pre-post factorial design: type of exercise (WIT $v s$. EFAM-UV ${ }^{\circ}$ ) and dose-distribution (CON $v s$. ACC) as main factors. This resulted in 4 groups of exercise: EFAM-UV ${ }^{\circ} \mathrm{CON}$, EFAM-UV ${ }^{\odot} A C C$, WIT-CON and WIT-ACC, who trained along 15 weeks $(2 \times 7$ weeks, interspersed with 1 week of rest at week 8). CON groups trained for $60 \mathrm{~min} / \mathrm{session}$ always in the morning and at the same schedule, whereas ACC groups got exactly the same dose and intensity of exercise but distributed twice a day (i.e. $30 \mathrm{~min}$ in the morning and 30 more in the afternoon).

Eighty-eight elderly people volunteered to participate. The intervention was conducted by the local authorities, the general practitioners at the primary health care center and a team of sport sciences researchers. Inclusion criteria were: 1) $\geq 65$ years old; 2) fit to participate in a regular exercise program according to the medical referral; 3 ) currently sedentary (no participation in a regular exercise program or intentional activities beyond normal daily hab- 
its within the previous 4 months); and 4) reporting a gait speed higher than $0.6 \mathrm{~m} / \mathrm{s}$. Exclusion criteria were: 1 ) presence of any disorder that would prevent the patient from being able to complete a training program; 2) missing 4 or more consecutive training sessions; and 3) adherence lower than $75 \%$ to the training sessions.

Since 31 individuals did not meet the inclusion criteria after the first screening, 57 sedentary older adults (31 female) completed the preintervention assessments ( 3 weeks) and initiated the training. They were homogeneously stratified in the 4 groups, in terms of age, gender, BMI and gait speed in $6 \mathrm{~m}$ (this last categorized according to the "Practical Guide for Prescribing a Multi-Component Physical Training Program to prevent weakness and falls in People over 70"). ${ }^{25}$ Finally, 46 participants finished the whole intervention: EFAM-UV ${ }^{\odot} \mathrm{CON}(\mathrm{N} .=15 ; 11$ female), EFAM-UV ${ }^{\circ} A C C(N .=11 ; 5$ female), WIT-CON $(\mathrm{N} .=11 ; 5$ female) and WIT-ACC (N. $=9$; 3 female). Table I summarizes their characteristics at baseline.

All individuals were previously informed and signed their written consent to participate in this study approved by the ethic committee of the University of Valencia. Health status and medications were stable during the intervention, as monitored by with doctors of the Local Health Centre, who collaborated in this research project (Table II).

Body composition and health control

Before and after the intervention, body composition was evaluated by bioimpedance (TANITA, model BC-545N, Tokyo, Japan), controlling the food intake and hydration in

TABLE I.-Description of sample at baseline (mean and standard deviation).

\begin{tabular}{|c|c|c|c|c|c|}
\hline \multirow{2}{*}{ Parameters } & \multirow{2}{*}{ Total $(\mathrm{N} .=46)$} & \multicolumn{2}{|c|}{ EFAM-UV@ $(\mathrm{N} .=26)$} & \multicolumn{2}{|c|}{ WIT $(\mathrm{N} .=20)$} \\
\hline & & Continuous (15) & Intermittent (11) & Continuous (11) & Intermittent (9) \\
\hline Age (years) & $71.8 \pm 3.8$ & $71.6 \pm 5.0$ & $72.9 \pm 3.7$ & $72.1 \pm 3.3$ & $70.4 \pm 4.1$ \\
\hline Weight (kg) & $73.9 \pm 13.0$ & $72.8 \pm 12.0$ & $71.2 \pm 15.6$ & $71.5 \pm 11.1$ & $82.2 \pm 11.9$ \\
\hline Height (cm) & $160.3 \pm 7.8$ & $159.7 \pm 8.6$ & $159.1 \pm 9.2$ & $159.9 \pm 6.8$ & $163.4 \pm 5.4$ \\
\hline BMI (index) & $28.6 \pm 3.5$ & $28.4 \pm 3.3$ & $27.8 \pm 3.5$ & $27.8 \pm 3.1$ & $30.7 \pm 4.0$ \\
\hline $\mathrm{FM}(\%)$ & $36.1 \pm 6.5$ & $38.3 \pm 6.4$ & $35.4 \pm 5.1$ & $34.6 \pm 7.3$ & $35.0 \pm 7.3$ \\
\hline FM (kg) & $26.6 \pm 6.5$ & $27.7 \pm 5.9$ & $25.1 \pm 5.8$ & $24.5 \pm 5.6$ & $29.2 \pm 8.9$ \\
\hline FFM (kg) & $47.3 \pm 9.9$ & $44.9 \pm 9.4$ & $46.1 \pm 11.4$ & $47.0 \pm 10.2$ & $53.0 \pm 7.1$ \\
\hline $\mathrm{SBP}(\mathrm{mmHg})$ & $150.4 \pm 18.7$ & $145.5 \pm 19.7$ & $155.6 \pm 17.0$ & $151.1 \pm 15.2$ & $149.0 \pm 17.9$ \\
\hline DBP (mmHg) & $82.0 \pm 10.3$ & $81.3 \pm 9.8$ & $83.0 \pm 9.7$ & $83.4 \pm 10.1$ & $80.6 \pm 12.4$ \\
\hline $\mathrm{SaO}_{2}(\%)$ & $95.7 \pm 3.5$ & $97.00 \pm 1.3$ & $97.0 \pm 1.3$ & $95.3 \pm 2.7$ & $93.1 \pm 6.5$ \\
\hline HR (bpm) & $73.0 \pm 9.8$ & $70.7 \pm 6.8$ & $74.2 \pm 11.9$ & $75.3 \pm 13.1$ & $71.8 \pm 10.1$ \\
\hline Gait speed (m/s) & $1.08 \pm 0.17$ & $1.00 \pm 0.2$ & $1.1 \pm 0.1$ & $1.2 \pm 0.2$ & $1.1 \pm 0.2$ \\
\hline \multicolumn{6}{|l|}{ Gender $(\%)$} \\
\hline Female & 52.4 & 66.7 & 45.5 & 45.5 & 22.2 \\
\hline Male & 47.8 & & 54.5 & 54.5 & 77.8 \\
\hline \multicolumn{6}{|l|}{ Education (\%) } \\
\hline No education & 17.4 & & 27.3 & 27.3 & 11.1 \\
\hline Primary School & 65.2 & & 63.6 & 63.6 & 88.9 \\
\hline Senior High Scho & 8.7 & & 9.1 & 9.1 & 0.0 \\
\hline University degree & 8.7 & 6.7 & 0.0 & 0.0 & 0.0 \\
\hline \multicolumn{6}{|c|}{ Economic status $(\%)$} \\
\hline Low income & 13.0 & 13.3 & 27.3 & 0.0 & 11.1 \\
\hline Middle income & 58.7 & 60.0 & 63.6 & 54.5 & 44.4 \\
\hline High income & 28.3 & 26.7 & 9.1 & 45.5 & 44.4 \\
\hline
\end{tabular}

BMI: Body Mass Index; FM: fat mass; SBP: systolic blood pressure; DBP: diastolic blood pressure; $\mathrm{SaO}_{2}$ : oxygen saturation; HR: heart rate.

TABLE II.-Experimental procedure.

\begin{tabular}{|c|c|c|c|}
\hline & Height & EFAM-UVCCON & Height \\
\hline & 1 month before & 1 week before & 1 week before \\
\hline \multirow[t]{3}{*}{ Socioeconomic questionnaire } & Body composition & EFAM-UVCACC & Body composition \\
\hline & Blood pressure & WITCON & Blood pressure \\
\hline & Heart rate $-\mathrm{SaO}^{2}$ & WITACC & Heart rate $-\mathrm{SaO}^{2}$ \\
\hline Gait speed (6 meters) & EQ-5D-5L - VIDA & & Gait speed (6 meters) \\
\hline Screening phase & Evaluation (Pre) & Intervention (15 weeks) & Evaluation (Post) \\
\hline
\end{tabular}


the previous hours to reproduce the evaluation conditions. Postintervention measurements were at least $48 \mathrm{~h}$ after exercise cessation, looking for proper rehydration.

Weight $(\mathrm{kg})$, fat mass (FM, \%), and the estimated lean mass $(\mathrm{kg})$ and bone mass $(\mathrm{kg})$ were registered from the Tanita, after measuring the height $(\mathrm{cm})$ by means of a stadiometer (SECA 222, Hamburg, Germany). Later on, according to a two-compartmental model with body divided in FM and fat-free mass (FFM), ${ }^{26}$ this FFM (kg) was calculated by adding the estimated lean mass and the bone mass. Body Mass Index (BMI), and FM (in kg) were also considered to characterize the sample.

Despite its limitations, ${ }^{26,27}$ the Bioelectrical Impedance Analysis has been gaining acceptance and validity in fields such as nutrition, gerontology and sports science, 28,29 and has been used in other body composition studies with similar samples. ${ }^{30}$ Bioelectrical impedance measurements are based in the analysis of the conductance of water, present only in the fat-free mass (FFT), so this technique is accurate in clinical practice if euhydration, height, weight and other variables are added and controlled. ${ }^{27}$

\section{Gait speed}

Gait speed (GS) was determined over ground on a 4.5 meters walkway, using a system of two electric photocells by means of the Chronojump Software (Velleman PEM10D photocell, Cronojump Bosco System, response time $5-100 \mathrm{~ms}$ ). The participants completed the distance (without acceleration but with a 2 meters deceleration zone) walking at a comfortable and usual pace, and the mean of three attempts was taken as GS. This GS was evaluated in the screening phase and after the intervention, to give light of some of its functional changes.

\section{Other variables}

Socio-economic data (level of studies, gender and economic satisfaction), biological data (age, blood pressure, oxygen saturation and heart rate) and psychosocial data (perceived quality of life and perceived autonomy) were collected also during the process of participants' selection in order to characterize the sample. For perceived quality of life and perceived autonomy, EuroQol 5D-5 $\mathrm{L}^{31}$ and VIDA questionnaire ${ }^{32}$ were used.

\section{Walking program}

Based on Malatesta et al., ${ }^{33}$ all the participants in the WIT program trained 3 times a week during the 15 weeks of training. Whilst Malatesta et al. ${ }^{33}$ programmed 7 weeks of WIT, increasing intensities from $50 \%$ to the $100 \%$ of the individual heart rate (HR) at the ventilatory threshold, intervals and intensities in our study were increased and scheduled considering the rating of perceived effort scale (RPE 1-10), with the value 7 (Very hard) representing the anaerobic threshold. 34,35

As shown in Figure 1, along the first seven weeks of WIT, we combined 2 to 4 min walking-hard intervals (increasing RPE from 3 to 7) with similar recovery periods of walking-moderate (RPE: 2 to 4), similar to Malatesta D, Simar D, Ben Saad H, Prefaut C and Caillaud C (33). After the control/resting $8^{\text {th }}$ week, intervals and intensities

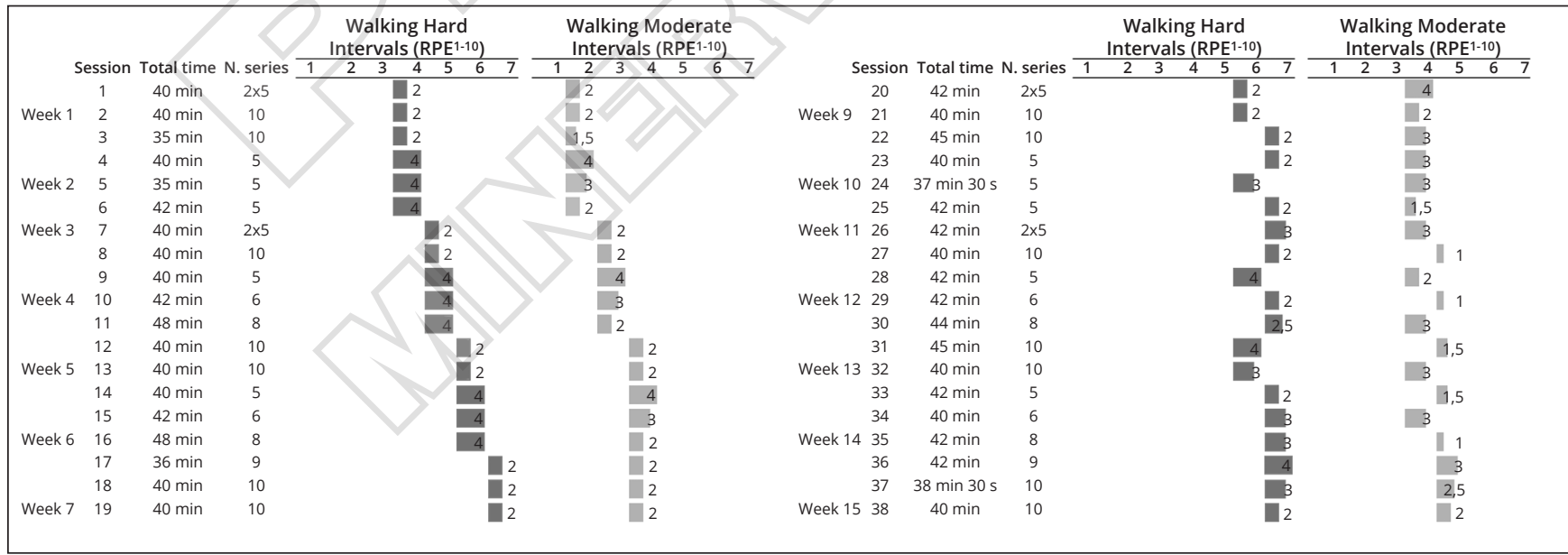

Figure 1.-Walking Interval Training program (WIT).

Numbers in the tittle row display RPE (Rating of Perceived Exertion, from 1 to 7 in the Borg Scale 1-10) as markers of the intervals' intensity in the walking intervention. Numbers in each cell indicates de interval duration for the walking hard (left column) and moderate (right column) intervals in each session. The intervention was periodized and divided in two blocks of 7 weeks, interspersed with one of testing (week 8). 
carried on increasing, staying close to the threshold intensity in the walking-hard (RPE: 6-7), and over the initial intensity in the walking-moderate intervals (RPE: 4-5). This progression lead participants to complete 7 trails of $3 \mathrm{~min}$ $(\mathrm{RPE} 7)+3 \min (\mathrm{RPE} 5)$ in the 36 session, the hardest in our schedule (Figure 1).

All sessions began with a brief warm-up period and ended with a cool down including breathing exercises, stability and joints mobility exercises. Participants were instructed to walk close to the programmed RPE, reinforcing the first sessions with some RPE familiarization tasks. In addition, in order to control the cardiac intensity (target: $\leq 80 \% \mathrm{HR}_{\max }$ ), they were provided with a Beurer PM-15 HR monitor, without chest strap (Beurer, Ulm, Germany), and one individualized card with the HR estimated for every Borg zone.

\section{EFAM-UV ${ }^{\circledR}$ multicomponent training program}

EFAM-UV ${ }^{024}$ is a neuromotor and cognitive MCT, based on dual-tasking, gait and postural control (re-)training, plus rhythm, and strength proposals. Under a comprehensive approach, training sessions integrate strength, mobility, motor-control, balance and rhythmic/aerobic exercises, evolving through the PE continuum from neuromuscular to bioenergetics demands, where executive function is a permanent target.

EFAM-UV@24 sessions usually start with postural control and gait-training tasks (motor coordination dual-tasks), sometimes enriched with cognitive constraints. Then it comes an increase in the intensity though: 1) strength/balance exercises with elastic bands or small dumbbells (in alternating days), combined with sticks and unstable material like the Fitball or sponge balls to go further; and 2) aerobic/rhythmic exercises, again in different days and depending on periodized objectives. The session concludes with social amusing tasks to cool down. ${ }^{24}$ In contrast to other MTC, EFAM-UV ${ }^{\odot}$ does not perform series in most of its neuromuscular proposals, since it tries to vary and enlarge the tasks/stimuli in every session.

Based on its authors, ${ }^{24}$ participants in EFAM-UV $\mathbb{C}$ groups trained twice a week, a frequency that is sufficient to improve physical and executive function in similar samples. ${ }^{36,37}$ We assumed these differences in frequency and total volume compared to WIT (Table III). ${ }^{24,36}$

Statistical analysis

Data were analyzed using the Statistical Package for the Social Sciences, SPSS v24 for Windows (IBM Inc. Chicago, IL, USA). After testing for normality (Shapiro-Wilk
Test), a Repeated Measures ANOVA was conducted to analyze changes in body composition considering: the main effect of the intervention (pre-post overall comparison); the type of exercise (WIT $v s$. EFAM-UV $\odot$ ); the dose-distribution (CON vs. ACC); and their interaction (type*dose-distribution). Within-Subjects Effects Tests at the first level, followed by Bonferroni post-hoc tests, were performed with statistical significance set at the level of $\mathrm{P} \leq 0.05$. Only when assessing the significance of differences in perceived autonomy and perceived quality of life between dropouts and the final sample, we had to use the non-parametric Mann-Whitney Test.

\section{Results}

Table I presents the description of the sample before the intervention, including body composition, health control variables and sociodemographic data. The mean age of the participants was $71.3 \pm 4.3$ years, classified as overweight,

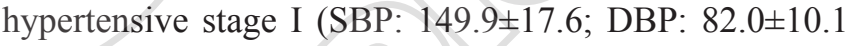
$\mathrm{mmHg}$ ) elderly. Regarding gait speed, all participants were able to walk above $1 \mathrm{~m} / \mathrm{s}$, a minimum speed to be considered independent and non-frail elderly adults. ${ }^{25} \mathrm{Im}$ portantly, intergroup comparisons at baseline showed no significant differences for any body composition outcome, nor for gait speed or age.

No-significant differences were found between dropouts and participants (Table IV), so no self-selection bias exists in the final sample.

Regarding the main effect of the intervention(Table V,VI), the repeated-measures ANOVA showed significant changes $(\mathrm{P}<0.005)$ in body composition and gait speed. The slight decrease in weight status $(73.948 \pm 12.433 \mathrm{~kg} v s .73 .102 \pm 12.174$ $\mathrm{kg})$ and BMI (28.574 \pm 3.526 vs. $28.160 \pm 3.323)$, was ac-

\begin{tabular}{|c|c|c|c|}
\hline \multicolumn{4}{|c|}{ TABLE III.-EFAM-UVO program. 24,36} \\
\hline \multicolumn{4}{|c|}{ Rough coordination $\rightarrow 4$ basic domains } \\
\hline $\begin{array}{l}\text { Postural control } \\
\text { (B) }\end{array}$ & $\begin{array}{l}\text { Gait patterns } \\
\text { (B) }\end{array}$ & $\begin{array}{l}\text { Manipulative } \\
\text { skills (C) }\end{array}$ & $\begin{array}{c}\text { Cognitive skills } \\
\text { (C) }\end{array}$ \\
\hline \multicolumn{4}{|c|}{ Adaptive precise coordination $\rightarrow 2$ complex domains } \\
\hline \multicolumn{2}{|c|}{ Rhythm } & \multicolumn{2}{|c|}{ Motor skills (functional) } \\
\hline \multicolumn{2}{|c|}{ Neuromuscular } & getic & Cognitive \\
\hline
\end{tabular}

EFAM-UV $\odot$ neuromotor training methodology aims to improve two basic skills (B) in the neuromotor re-training of the elderlies: postural control and gait patterns. Manipulative and cognitive skills are also included as complementary domains (C) in a primary level of training (i.e. rough coordination). Once the technical dexterity allows it, technicians introduce rhythm and motor skills tasks (complex domains) to increase the demands on executive function and motor control, looking for a more adaptive and precise coordination. Along with the increase in the task complexity, EFAM-UVO also sets methodological guidelines to increase the load through the three main directions of the re-training/learning process (bioenergetics, neuromuscular and cognitive). 
companied by positive changes in body composition: FM $(36.083 \pm 6.498 \%$ vs. $31.813 \pm 7.898 \%)$, FFM $(47.781 \pm 1.465$ $\mathrm{kg} v s .50 .726 \pm 1.537 \mathrm{~kg}$ ), jointly with an improvement in GS $(1.095 \pm 0.177 \mathrm{~m} / \mathrm{s}$ vs. $1.451 \pm 0.203 \mathrm{~m} / \mathrm{s})$. According to the partial eta square, this effect was moderate on FM, FFM and a bit bigger for GS.

The Within-Subjects Effects Test also showed a significant main effect of "type of exercise" $(\mathrm{P}<0.005)$ on FM $(\%), F M(\mathrm{~kg}), \mathrm{FMM}$, and GS, with half the effect size.

TABLE IV.-Differences between dropouts and final sample at baseline.

\begin{tabular}{lccc}
\hline & Dropouts $(\mathrm{N} .=11)$ & Participants $(\mathrm{N} .=46)$ & $\mathrm{P}$ \\
\hline EQindex & $0.678 \pm 0.089$ & $0.844 \pm 0.020$ & 0.074 \\
EQVAS & $74.833 \pm 4.267$ & $76.174 \pm 2.385$ & 0.727 \\
VIDA & $32.917 \pm 0.783$ & $32.848 \pm 0.375$ & 0.985 \\
\hline
\end{tabular}

P: statistical differences by Mann-Whitney U-Test. Significance level $\mathrm{P}<0.05$. EQindex: Descriptive item from the EQ-5D-5L; EQVAS: EQ Visual Analogue Scale; VIDA: Questionnaire for Assessing Instrumental Activities of Daily Living In Elderly People.

TABLE V.-Tests of within-subjects effects.

\begin{tabular}{|c|c|c|c|c|c|c|}
\hline Variable & $\begin{array}{l}\text { Type III } \\
\text { sum of } \\
\text { squares }\end{array}$ & df & $\begin{array}{l}\text { Mean } \\
\text { square }\end{array}$ & $\mathrm{F}$ & & $\begin{array}{c}\text { Partial } \\
\text { eta } \\
\text { square }\end{array}$ \\
\hline \multicolumn{7}{|l|}{ Intervention } \\
\hline Weight & 19.102 & 1 & 19.102 & 12.917 & $0.001 *$ & 0.235 \\
\hline BMI & 4.871 & 1 & 4.871 & 9.098 & $0.004 *$ & 0.178 \\
\hline FM (\%) & 462.989 & 1 & 462.989 & 69.179 & $0.001 *$ & 0.622 \\
\hline FM (kg) & 327.174 & 1 & 174.947 & 79.629 & $0.001 *$ & 0.655 \\
\hline FFM (kg) & 192.917 & 1 & 192.917 & 57.788 & $0.001 *$ & 0.579 \\
\hline GS (m/s) & 2.782 & 1 & 2.782 & 192.496 & $0.001 *$ & 0.821 \\
\hline \multicolumn{7}{|c|}{ Intervention $*$ type of exercise } \\
\hline Weight & 0.421 & 1 & & 0.285 & 0.597 & \\
\hline BMI & 0.888 & & 0.888 & 1.658 & 0.205 & 0.038 \\
\hline FM (\%) & 107.153 & 1 & 107.153 & 16.011 & $0.001 *$ & 0.276 \\
\hline FM (kg) & 51.291 & & 51.291 & 15.364 & $0.001 *$ & 0.268 \\
\hline FFM (kg) & 76.245 & 1 & 76.245 & 18.557 & $0.001 *$ & 0.306 \\
\hline $\mathrm{GS}(\mathrm{m} / \mathrm{s})$ & 0.183 & 1 & 0.183 & 12.657 & $0.001 *$ & 0.232 \\
\hline \multicolumn{7}{|c|}{ Intervention * dose distribution } \\
\hline Weight & 3.744 & 1 & 3.744 & .532 & 0.119 & 0.057 \\
\hline BMI & 0.662 & 1 & 0.662 & 1.236 & 0.273 & 0.029 \\
\hline $\mathrm{FM}(\%)$ & 3.153 & 1 & 3.153 & 0.471 & 0.496 & 0.011 \\
\hline FM (kg) & 10.197 & 1 & 10.197 & 2.482 & 0.870 & 0.056 \\
\hline FFM (kg) & 0.091 & 1 & 0.091 & 0.027 & 0.870 & 0.001 \\
\hline GS (m/s) & 0.003 & 1 & 0.003 & 0.198 & 0.658 & 0.005 \\
\hline \multicolumn{7}{|c|}{ Intervention * type of program * Dose distribution } \\
\hline Weight & 1.065 & 1 & 1.065 & 0.720 & 0.401 & 0.017 \\
\hline BMI & 0.204 & 1 & 0.204 & 0.381 & 0.541 & 0.009 \\
\hline FM $(\%)$ & 21.769 & 1 & 21.769 & 3.253 & $0.078^{\dagger}$ & 0.072 \\
\hline FM (kg) & 12.679 & 1 & 12.679 & 3.086 & $0.086^{\dagger}$ & 0.068 \\
\hline FFM (kg) & 23.032 & 1 & 23.032 & 6.899 & $0.012 *$ & 0.141 \\
\hline $\mathrm{GS}(\mathrm{m} / \mathrm{s})$ & 0.007 & 1 & 0.007 & 0.470 & 0.497 & 0.011 \\
\hline
\end{tabular}

BMI: Body Mass Index; BF: body fat; FM: fat mass; FFM: free fat mass; GS:

gait speed.
$*$ Significance level $\mathrm{P}<0.050$; ${ }^{\dagger}$ significance level $\mathrm{P}<0.100$.
TABLE VI.-Mean and SD of pre-post changes when considering whole sample and the levels of type of exercise (MCTP and WP), and dose-distribution (continuous and accumulated).

\begin{tabular}{|c|c|c|c|c|c|c|c|c|}
\hline \multirow{2}{*}{ Variable } & \multirow{2}{*}{ N. } & \multicolumn{2}{|c|}{ Baseline } & \multicolumn{2}{|c|}{ Post } & \multirow{2}{*}{$\Delta \%$} & \multirow{2}{*}{$\mathrm{P}$} & \multirow{2}{*}{ ES } \\
\hline & & Mean & SD & Mean & SD & & & \\
\hline \multicolumn{9}{|l|}{ All } \\
\hline FM (\%) & 46 & 36.1 & 6.5 & 31.8 & 7.9 & -11.8 & $0.001 *$ & 0.6 \\
\hline FFM (kg) & 46 & 47.3 & 9.9 & 50.1 & 10.9 & 5.9 & $0.001 *$ & 0.3 \\
\hline \multicolumn{9}{|l|}{ EFAM-UV৫ } \\
\hline FM (\%) & 26 & 37.1 & 5.9 & 34.6 & 6.3 & -6.6 & $0.002 *$ & 0.4 \\
\hline FFM (kg) & 26 & 45.4 & 10.1 & 47.0 & 9.7 & 3.5 & $0.008 *$ & 0.2 \\
\hline \multicolumn{9}{|l|}{ WIT } \\
\hline FM (\%) & 20 & & 7.1 & 28.2 & 8.4 & -19.0 & $0.001 *$ & 0.8 \\
\hline FFM (kg) & 20 & & 9.3 & 54.1 & 11.3 & 8.8 & $0.001 *$ & 0.4 \\
\hline \multicolumn{9}{|l|}{ Continuous } \\
\hline FM (\%) & 26 & 36.7 & 6.9 & 32.7 & 7.6 & -10.9 & $0.001 *$ & 0.6 \\
\hline FFM (kg) & 26 & 45.8 & 9.6 & 48.6 & 9.9 & 6.1 & $0.001 *$ & 0.3 \\
\hline \multicolumn{9}{|c|}{ Accumulated } \\
\hline FM (\%) & 20 & 35.2 & 6.0 & 30.6 & 8.3 & -13.1 & $0.001 *$ & 0.6 \\
\hline FFM (kg) & 20 & 49.3 & 10.1 & 52.0 & 12.0 & 5.6 & $0.001 *$ & 0.2 \\
\hline
\end{tabular}

FM: Fat mass; FFM: free fat mass; $\Delta \%$ : pre-post percentage of change; ES: effect size (d).

*Significance level $\mathrm{P}<0.050$; ${ }^{\dagger}$ significance level $\mathrm{P}<0.100$.

But there was a moderating effect of the "dose-distribution" strategy, only on the body composition, as revealed by a significant interactive effect of both factors on FFM $(\mathrm{P}=0.012)$, and a trend to significance $(\mathrm{P}<0.10)$ on $\mathrm{FM}(\%)$ and FM(kg) (Table V, VI).

Further Bonferroni analyses of pre-post differences for each type*dose combination showed significant improvements except for EFAM-UV ${ }^{\odot} \mathrm{ACC}$ on FM and FFM (Figure 2).

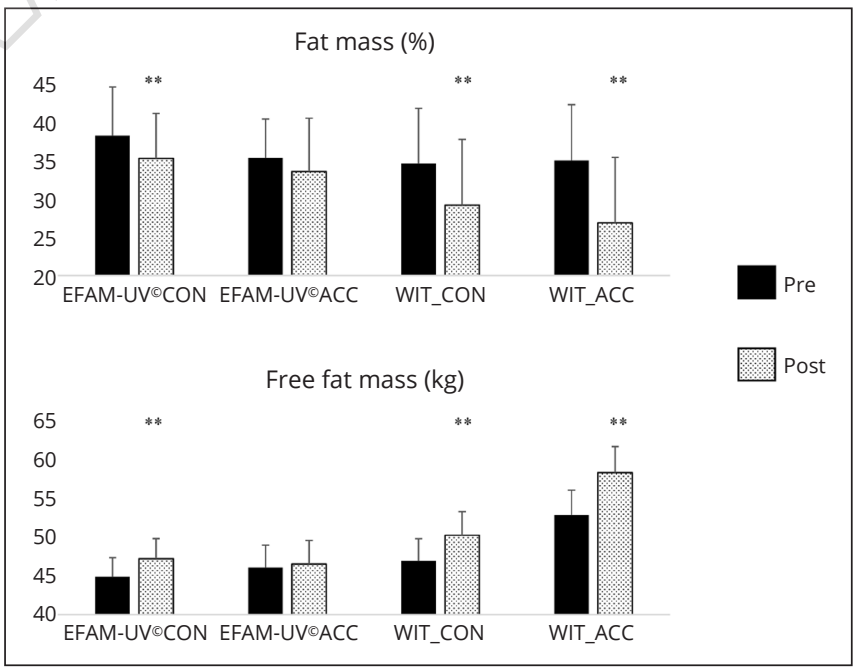

Figure 2.-Bonferroni post-hoc comparisons between type of exercise and dose-distribution strategy.

$* \mathrm{P}<0.050 ; * * \mathrm{P}<0.010$. 


\section{Discussion}

Although the positive effect of breaking the sedentary time with brief bouts of exercise has been widely demonstrated, as far as we know this is the first study to compare the effect of distributing or concentrating the dose of two types of periodized exercise on the body composition of sedentary elders. Specifically, the study analyses the effect of a multicomponent training program and a walking intervaltraining program (both respecting their authors' guidelines) comparing the effect of continuous $v s$. accumulated dose distributions. According to our results, besides enhancing functionality, tailored and properly periodized PE improves body composition in sedentary elderly regardless the training strategy (type of exercise or dose distribution). Moreover, whilst the manipulation of the dose over daytime, seems not to exert differential effects on body composition per se, the type of exercise has a medium effect on fat a lean body mass, which is moderated by the dose strategy.

Despite the medium duration of the intervention, ${ }^{38}$ our data confirm the positive impact of starting PE when it is properly programmed, tailored and supervised. 19,39 The significant reduction of a $11.8 \% \mathrm{FM} \%$ and the gain of $5.9 \%$ FFM (muscular and bone tissue) may explain in part the improvement in GS, probably contributing to enhance in the short term the health status and health-related quality of life of this population. ${ }^{17,40}$

When analyzing FM and FFM in both PE programs separately, WIT confirmed its metabolic effect, with larger values as compared to EFAM-UV ${ }^{\circ}(19$ vs. $6.6 \%$ reduction in FM\%, $8.8 v$ s. 3.5\% gain in FFM), and also larger effect size ( 0.8 vs. 0.4 for $\mathrm{FF} \% ; 0.4$ vs. 0.2 for FFM). The meta-analysis by Hanson and Jones ${ }^{40}$ displayed a mean effect size of -1.31 in the FM\% after walking interventions, but our intervention was addressed to sedentary elderly and lasted only 15 weeks. With regard to EFAM-UV ${ }^{\circ}$, the fact of getting positive changes in body composition in this short period, and with only two sessions a week. This confirms the positive effect of comprehensive programs which combine strength and cardiovascular demands, despite the predominance of the former and its neuromuscular approach. We are far, for example, from the big reduction in $\mathrm{FM} \%$ by Leitão et al. 30,41 ( -16.5 and $-15.17 \mathrm{FM} \%$ in their studies, with samples similar to ours, and an effect size over 2), but again, their intervention lasted 36 weeks (2.5-fold our volume of training). Importantly, BMI and weight improved only when considering the main effect across intervention groups, what may be related to its bigger sample. Notwithstanding, BMI and weight do not distinguish between FM and FFM and they may not fully reflect changes in body composition with aging. ${ }^{42}$
As above mentioned, there was no significant effect when comparing the manipulation of the dose (CON vs. ACC) so the strategy $\mathrm{x}$ dose interaction will be interpreted in terms of moderation of strategy effects by dose (i.e., the interaction of the two strategies, Figure 2). FM and FFM improved in three of them (WIT-CON, WIT-ACC $\&$ EFAM-UV $\left.{ }^{\circ} \mathrm{CON}\right)$, and these beneficial effects were of limited and negligible size when distributing the multicomponent training over daytime (EFAM-UV ${ }^{\circ}$ ACC).

Cardiovascular walking programs have previously shown a certain consistency in relation to the improvements in FM. ${ }^{40,} 43$ They ensure enough metabolic stimuli whatever the dose distribution, whilst these demands might be lower in MCT programs, which typically prioritize neuromuscular training over metabolic stimulation. In addition, differences in the manipulation of parameters such as dose or intensity, and the more complex nature of the tasks in any multicomponent training, explain some inconsistencies in the results of this type of programs, also with regard body composition of sedentary elders. ${ }^{9}$ For example, Marques et al. ${ }^{44}$ did not find differences in body fat after 8 months of exercise (2 days/week) in elderlies who underwent a resistance program vs. a multicomponent program).

Importantly, although differences in total volume and nature of the tasks strongly limit the comparability, EFAM$\mathrm{UV}^{\odot} \mathrm{CON}$ reduced FM with only 2 days/week compared to the 3 days/week of the WIT programs. EFAM-UV ${ }^{\odot}$ is always periodized to initially accomplish neuromuscular goals (strength and balance in the first mesocycles) and move on to increased cardiovascular demands at the end of the macrocycle, when the elderly individuals are able to perform them under safe conditions. The introduction of rhythm and motor skill tasks also leads to increase cardiovascular demands and fun at the end of any intervention. Optimizing the time of exercise through continuous changes in affordable tasks is another important target in the program, so 60 min may ensure reaching the metabolic demands necessary to impact body composition. Conversely, distributing this dose seems to reduce these benefits.

Although we have not measured sedentary time and daily PA, the increase in physically active time and social interaction due to the participation in the exercise intervention, jointly with enhanced physical function following the intervention, may have helped to break sedentary lifestyle, which is considered the main contributor to the increase weight at these ages. ${ }^{45}$

Of interest, the same three experimental groups (WITCON, WIT-ACC and EFAM-UV ${ }^{\circ} \mathrm{CON}$ ) have been effective to increase the FFM, confirming the intervention suit- 
ability and effectiveness on lean mass and muscle mass. With regard to this former, it stands out that most studies based on walking programs do not achieve significant increases in muscle mass, at least at a general level; $4,43,46$ and in turn, multicomponent programs have shown inconsistent results. The review of Marín-Cascales et al. ${ }^{47}$ points out that changes in lean body mass after multicomponent physical interventions are also unclear. Walking is one of the most frequent types of aerobic activity used in multicomponent training. So, it would be important to achieve at least 30 min of aerobic walking exercise, combined with strength, agility, coordination or breathing tasks, ${ }^{47}$ to ensure the minimum intensity to increase lean body mass in the elderly participants. In fact Aragão et al. ${ }^{3}$ showed a significant increase in muscle mass following 12 months of a multicomponent program (3 days/week) in postmenopausal women despite they usually experience a progressive reduction of lean body mass in absence of physical exercise. Although the bioimpedance analysis only estimates lean mass and bone mass from the FFM, the outcomes of the present study suggest that 2 days per week of MCT may improve this component of the FFM, but only after 60 min of continuous training (EFAM-UV $\left.{ }^{\circ} \mathrm{CON}\right)$. Instead, distributing the volume/stimuli in doses of $30 \mathrm{~min}$ in the morning and $30 \mathrm{~min}$ in the afternoon (EFAM-UV॰ACC) failed to induce similar adaptations, at least after the 15 weeks of training.

A similar rationale would suggest some bone benefits after WIT-CON, WIT-ACC and EFAM-UV ${ }^{\odot} \mathrm{CON}$, since the three of them include walking tasks and osteoarticular impact. In light of previous studies, the increase in bone mass through WP remains still controversial, although they have confirmed to reduce or even stop its progressive loss. ${ }^{2}$ The intensity of walking is an important matter, ${ }^{48}$ so the gradual increase in intensity and the modulation of the lap-duration through the whole WIT might have helped. Hatori et al. ${ }^{48}$ compared walking above (high-intensity) or below (low-intensity) the anaerobic threshold and pointed out that the lowintensity group and controls showed a similar loss of bone density, whereas the high-intensity group showed improvements. Walking intensities close to this threshold (RPE of 7) in the last weeks of the present intervention might derive in an increase in bone mass. On the other hand, MCT has been proposed as one of the most recommended intervention to stop the loss of bone mass during aging, ${ }^{47}$ although given the variability of the protocols and outcome measures, the results of these methods are difficult to quantify. ${ }^{49}$ Future studies with specific densitometry technologies should be conducted to confirm this encouraging expectative in EFAM-UV ${ }^{\odot} \mathrm{CON}$, since it is performed only twice a week.
Hence, our data confirm that starting physical exercise through periodized, individualized and supervised programs, guided and tailored for elderly population, is effective to improve their body composition regardless the type of exercise. ${ }^{9}$ The combination of sarcopenia with increased FM generates a reduction in cardiovascular fitness; and the reduction of bone mass leads to increase risk of falls and comorbidity. Since all of these detrimental changes are agerelated and accelerated by sedentary lifestyle, WIT-CON, WIT-ACC and EFAM-UV ${ }^{\odot} \mathrm{CON}$ might contribute to preserve bone mass, muscle mass, and potentially reduce risk of fall, comorbidity and all-cause mortality in sedentary elderly. ${ }^{1}$ Our data suggest that the accumulated strategy in the multicomponent approach (EFAM-UV $\left.{ }^{\circ} \mathrm{ACC}\right)$ will probably need some more time to induce similar benefits on elderlies' body composition. Again this warrants further research, testing longer intervention periods.

Previous reviews ${ }^{40,50}$ already pointed that aerobic conditioning programs (i.e. walking) could be a key element in the design of weight control strategies in older adults, although multicomponent programs generally have shown greater benefits at the functional level. ${ }^{22}$ With regard to dose distribution, both strategies could induce different adaptations due to differences in the effective intensity of exercise. Concentrating the load may increase fatigue and lead to more pronounced physiological alterations, ${ }^{51}$ so continuous dose would lead to higher exertion for exercises with similar external intensity. Actually, the beneficial effects of both strategies (continuous $v s$. accumulated) have been demonstrated after 9 and 18 months of brisk walking in middle-age obese females ${ }^{52}$ where concentrated groups showed slightly better, although not significant, losses of body weight and specifically of the FM parameter.

Public health policies aimed to prescribe exercise must account for the impact of different strategies, with special attention to differences in the population at the short and the long term. In order to induce short-term changes in body composition, and address problems such as sarcopenia or dyslipidemia, continuous strategies might be more effective. Meanwhile, accumulated strategies may require a little more time. Further studies will elucidate whether longer accumulated interventions might help through changes in those sedentary behaviors that increase throughout the day. ${ }^{15}$

\section{Limitations of the study}

The small size of the sample when setting four groups implicates some uncertainty when extrapolating the results and becomes a limitation to our study. Future studies should seek to include a greater sample size to better elu- 
cidate mechanisms of exercise on body composition and physical function in relation to continuous versus accumulated dose. Besides, we did not include a control group who did not exercise. This was intentional from an ethical point of view because we considered the evidence of physical exercise impact on physical function in sedentary older adults. However, the major limitation of this study is the different weekly frequency of sessions in the WIT and EFAM-UV ${ }^{\odot}$ groups. It is possible that 15 weeks training in EFAM-UV ${ }^{\odot} \mathrm{ACC}$ condition would have been enough to elicit significant body composition changes if the frequency were 3 times per week as WIT. However, we have followed the authors' recommendations in both programs from a perspective of ecological validity.

\section{Conclusions}

Starting physical practice in sedentary older adults improves body composition regardless of the type of program performed and, in particular, EFAMUV ${ }^{\circ}$ gets improvements with only 2 days/week. The distribution strategy only gave positive results in the walking programs groups. Consequently, it is important that the institutions of prevention policies recognize the impact of different strategies for the short and long term. Concentrated strategies would be more recommendable to produce rapid changes in body composition, Notwithstanding, accumulated strategies may also have positive consequences, helping to change sedentary behavior despite of requiring some more time (especially in multicomponent programs).

\section{References}

1. Santanasto AJ, Goodpaster BH, Kritchevsky SB, Miljkovic I, Satterfield S, Schwartz AV, et al. Body Composition Remodeling and Mortality: The Health Aging and Body Composition Study. J Gerontol A Biol Sci Med Sci 2017;72:513-9.

2. Gómez-Cabello A, Ara I, González-Agüero A, Casajús JA, VicenteRodríguez G. Effects of training on bone mass in older adults: a systematic review. Sports Med 2012;42:301-25.

3. Aragão FR, Abrantes CG, Gabriel RE, Sousa MF, Castelo-Branco $\mathrm{C}$, Moreira MH. Effects of a 12-month multi-component exercise program on the body composition of postmenopausal women. Climacteric 2014;17:155-63.

4. Beavers KM, Ambrosius WT, Rejeski WJ, Burdette JH, Walkup MP, Sheedy JL, et al. Effect of Exercise Type During Intentional Weight Loss on Body Composition in Older Adults with Obesity. Obesity (Silver Spring) 2017;25:1823-9.

5. Beaudart C, Dawson A, Shaw SC, Harvey NC, Kanis JA, Binkley N, et al.; IOF-ESCEO Sarcopenia Working Group. Nutrition and physical activity in the prevention and treatment of sarcopenia: systematic review. Osteoporos Int 2017;28:1817-33.

6. Denison HJ, Cooper C, Sayer AA, Robinson SM. Prevention and optimal management of sarcopenia: a review of combined exercise and nu- trition interventions to improve muscle outcomes in older people. Clin Interv Aging 2015;10:859-69.

7. Lambert M. Over diagnosing? Time for the 'exercise is medicine'movement to react. S Afr J Sports Med 2015;27:62.

8. Murphy MH, Blair SN, Murtagh EM. Accumulated versus continuous exercise for health benefit: a review of empirical studies. Sports Med 2009;39:29-43.

9. Bouaziz W, Lang PO, Schmitt E, Kaltenbach G, Geny B, Vogel T. Health benefits of multicomponent training programmes in seniors: a systematic review. Int J Clin Pract 2016;70:520-36.

10. WHO. Recomendaciones mundiales sobre la actividad física para la salud. Geneva: WHO; 2010.

11. Aggio DA, Sartini C, Papacosta O, Lennon LT, Ash S, Whincup PH, et al. Cross-sectional associations of objectively measured physical activity and sedentary time with sarcopenia and sarcopenic obesity in older men. Prev Med 2016;91:264-72.

12. Haskell WL, Lee IM, Pate RR, Powell KE, Blair SN, Franklin BA, et al.; American College of Sports Medicine; American Heart Association. Physical activity and public health: updated recommendation for adults from the American College of Sports Medicine and the American Heart Association. Circulation 2007;116:1081-93.

13. Dempsey PC, Owen N, Biddle SJ, Dunstan DW. Managing sedentary behavior to reduce the risk of diabetes and cardiovascular disease. Curr Diab Rep 2014;14:522.

14. Healy GN, Dunstan DW, Salmon J, Cerin E, Shaw JE, Zimmet PZ, et al. Breaks in sedentary time: beneficial associations with metabolic risk. Diabetes Care 2008;31:661-6.

15. Sartini C, Wannamethee SG, Iliffe S, Morris RW, Ash S, Lennon L, et al. Diurnal patterns of objectively measured physical activity and sedentary behaviour in older men. BMC Public Health 2015;15:609.

16. Gallagher NA, Clarke P, Carr E. Physical activity in older adults in a combined functional circuit and walking program. Geriatr Nurs 2016;37:353-9.

17. Floegel TA, Giacobbi PR Jr, Dzierzewski JM, Aiken-Morgan AT, Roberts B, McCrae CS, et al. Intervention markers of physical activity maintenance in older adults. Am J Health Behav 2015;39:487-99.

18. Rosenberg DE, Kerr J, Sallis JF, Norman GJ, Calfas K, Patrick K. Promoting walking among older adults living in retirement communities. J Aging Phys Act 2012;20:379-94.

19. Mandini S, Collini G, Grazzi G, Lavezzi E, Mazzoni G, Conconi F. Reduction in risk factors for cardiovascular diseases and long-lasting walking habit in sedentary male and female subjects following 1 year of guided walking. Sport Sci Health 2018;14:121-6.

20. Murtagh EM, Nichols L, Mohammed MA, Holder R, Nevill AM, Murphy MH. The effect of walking on risk factors for cardiovascular disease: an updated systematic review and meta-analysis of randomised control trials. Prev Med 2015;72:34-43.

21. Maki Y, Ura C, Yamaguchi T, Murai T, Isahai M, Kaiho A, et al. Effects of intervention using a community-based walking program for prevention of mental decline: a randomized controlled trial. J Am Geriatr Soc 2012;60:505-10

22. Cadore EL, Rodríguez-Mañas L, Sinclair A, Izquierdo M. Effects of different exercise interventions on risk of falls, gait ability, and balance in physically frail older adults: a systematic review. Rejuvenation Res $2013 ; 16: 105-14$

23. Blasco-Lafarga C, Sanchis-Sanchis R, Sanchis-Soler G, San Inocencio-Cuenca D, Llorens P. Entrenamiento Neuromotor en pacientes ancianos pluripatológicos en las Unidades de Hospitalización a Domicilio: estudio. Cuad Psicol Deporte 2019;19:95-105.

24. Blasco-Lafarga C, Martinez-Navarro I, Cordellat A, Roldán A, Monteagudo P, Sanchis-Soler G, et al. Método de Entrenamiento Funcional Cognitivo Neuromotor. Propiedad Intelectual $n^{\circ} 156069$ 2016; España (2016).

25. Izquierdo M, Casas-Herrero A, Zambom-Ferraresi F, Martínez-Velilla N, Alonso-Bouzon C. Guía práctica para la prescripción de un programa 
de entrenamiento físico multicomponente para la prevención de la fragilidad y caídas en mayores de 70 años. En Representación de VIVIFRAIL; 2017.

26. Tinsley GM, Graybeal AJ, Moore ML, Nickerson BS. Fat-free Mass Characteristics of Muscular Physique Athletes. Med Sci Sports Exerc 2019;51:193-201.

27. Andreoli A, Garaci F, Cafarelli FP, Guglielmi G. Body composition in clinical practice. Eur J Radiol 2016;85:1461-8.

28. Jaffrin MY. Body composition determination by bioimpedance: an update. Curr Opin Clin Nutr Metab Care 2009;12:482-6.

29. Macfarlane DJ, Chan NT, Tse MA, Joe GM. Agreement between bioelectrical impedance and dual energy X-ray absorptiometry in assessing fat, lean and bone mass changes in adults after a lifestyle intervention. J Sports Sci 2016;34:1176-81.

30. Leitão L, Pereira A, Mazini M, Venturini G, Campos Y, Vieira J, et al. Effects of Three Months of Detraining on the Health Profile of Older Women after a Multicomponent Exercise Program. Int J Environ Res Public Health 2019;16:3881.

31. Herdman M, Gudex C, Lloyd A, Janssen M, Kind P, Parkin D, et al. Development and preliminary testing of the new five-level version of EQ5D (EQ-5D-5L). Qual Life Res 2011;20:1727-36.

32. Martin-Lesende I, Vrotsou K, Vergara I, Bueno A, Diez A. Design and validation of the vida questionnaire, for assessing instrumental activities of daily living in elderly people. J Gerontol Geriatr Res 2015;4:2.

33. Malatesta D, Simar D, Ben Saad H, Préfaut C, Caillaud C. Effect of an overground walking training on gait performance in healthy $65-$ to 80-year-olds. Exp Gerontol 2010;45:427-34.

34. Borg E, Kaijser L. A comparison between three rating scales for perceived exertion and two different work tests. Scand J Med Sci Sports 2006; 16:57-69.

35. Hill DW, Cureton KJ, Grisham SC, Collins MA. Effect of training on the rating of perceived exertion at the ventilatory threshold. Eur J App Physiol Occup Physiol 1987;56:206-11.

36. Blasco-Lafarga C, Monteagudo $\mathrm{P}$, Blasco-Lafarga N, Cordellat A Roldán A. Función ejecutiva, capacidad cardiovascular y calidad de vida en mayores del entorno rural: impacto de un programa multidisciplinar. Comunidad 2016;18:1-6.

37. Roldán A, Cordellat A, Monteagudo P, García-Lucerga C, BlascoLafarga NM, Gomez-Cabrera MC, et al. Beneficial Effects of Inspiratory Muscle Training Combined With Multicomponent Training in Elderly Active Women. Res Q Exerc Sport 2019;90:547-54.

38. Mian OS, Baltzopoulos V, Minetti AE, Narici MV. The impact of physical training on locomotor function in older people. Sports Med 2007;37:683-701.

39. Knight E, Stuckey MI, Petrella RJ. Prescribing physical activity through primary care: does activity intensity matter? Phys Sportsmed 2014;42:78-89.
40. Hanson S, Jones A. Is there evidence that walking groups have health benefits? A systematic review and meta-analysis. Br J Sports Med 2015;49:710-5.

41. Leitão L, Brito J, Leitão $\mathrm{A}$, Pereira $\mathrm{A}$, Conceição $\mathrm{A}$, Silva $\mathrm{A}$, et al. Functional capacity retention in older women after multicomponent exercise cessation: 3-year longitudinal study. Motricidade 2015;11:81-91.

42. Kahn HS, Cheng YJ. Comparison of adiposity indicators associated with fasting-state insulinemia, triglyceridemia, and related risk biomarkers in a nationally representative, adult population. Diabetes Res Clin Pract 2018;136:7-15.

43. Gába A, Cuberek R, Svoboda Z, Chmelík F, Pelclová J, Lehnert M, et al. The effect of brisk walking on postural stability, bone mineral density, body weight and composition in women over 50 years with a sedentary occupation: a randomized controlled trial. BMC Womens Health 2016;16:63.

44. Marques E, Carvalho J, Soares JM, Marques F, Mota J. Effects of resistance and multicomponent exercise on lipid profiles of older women. Maturitas 2009;63:84-8.

45. Gomez-Cabello A, Pedrero-Chamizo R, Olivares PR, Luzardo L, Juez-Bengoechea A, Mata E, et al.; EXERNET Study Group. Prevalence of overweight and obesity in non-institutionalized people aged 65 or over from Spain: the elderly EXERNET multi-centre study. Obes Rev 2011;12:583-92.

46. Karstoft K, Winding K, Knudsen SH, Nielsen JS, Thomsen C, Pedersen BK, et al. The effects of free-living interval-walking training on glycemic control, body composition, and physical fitness in type 2 diabetic patients: a randomized, controlled trial. Diabetes Care 2013;36:228-36.

47. Marín-Cascales E, Alcaraz PE, Ramos-Campo DJ, Rubio-Arias JA Effects of multicomponent training on lean and bone mass in postmenopausal and older women: a systematic review. Menopause 2018;25:346-56.

48. Hatori M, Hasegawa A, Adachi H, Shínozaki A, Hayashi R, Okano H, et al. The effects of walking at the anaerobic threshold level on vertebral bone loss in postmenopausal women. Calcif Tissue Int 1993;52:411-4.

49. Benedetti MG, Furlini G, Zati A, Letizia Mauro G. The Effectiveness of Physical Exercise on Bone Density in Osteoporotic Patients. BioMed Res Int 2018;2018:4840531.

50. Bouaziz W, Vogel T, Schmitt E, Kaltenbach G, Geny B, Lang PO. Health benefits of aerobic training programs in adults aged 70 and over: a systematic review. Arch Gerontol Geriatr 2017;69:110-27.

51. Campbell L, Wallman $K$, Green $D$. The effects of intermittent exercise on physiological outcomes in an obese population: continuous versus interval walking. J Sports Sci Med 2010;9:24-30.

52. Donnelly JE, Jacobsen DJ, Heelan KS, Seip R, Smith S. The effects of 18 months of intermittent vs. continuous exercise on aerobic capacity, body weight and composition, and metabolic fitness in previously sedentary, moderately obese females. Int J Obes Relat Metab Disord 2000;24:566-72.

Conflicts of interest.-The authors certify that there is no conflict of interest with any financial organization regarding the material discussed in the manuscript. Funding.- This work was part supported by a predoctoral grant of Conselleria d'Investigació de la Generalitat Valenciana [ACIF/2016/423]; and by the European Social Fund [BEFPI-2018].

Authors' contributions. - Cristina Blasco-Lafarga: conceived the study and coordinated the research; participated in the design of the test, the data collection along the experimental procedure, the design of statistical analysis, and finally drafted the manuscript; Pablo Monteagudo: conceived the study and coordinated the research; participated in the design of the intervention, the data collection along the experimental procedure, the design of statistical analysis, and finally drafted the manuscript; Ainoa Roldan: participated in the data collection along the experimental procedure; helped with the 16 collection of references and review of the draft; Ana Cordellat: participated in the data collection along the experimental procedure; helped with the statistical analysis, collection of references and review of the draft; Caterina Pesce: carried on the statistical analysis and helped with the collection of references, writing and review of the draft.

Congresses.-1) I International Congress of Physical Activity and Sports Sciences "Current and Future Challenges of Physical Activity and Sport" (UCV, Valencia, Spain, 2019); 2) XXXVI Congresso Nazionale FMSI (Rome, Italy, 2019).

Acknowledgements.-The authors want to thank the Buñol local politicians and the University of Valencia for their support, as well as the elderly association "Entrenamiento con Mayores".

History.-Article first published online: script received: August 30, 2019. 